\title{
Further Results on Local Stability of REM Algorithm with Time-Varying Delays
}

\author{
Huijun Gao, James Lam, Changhong Wang, and Xinping Guan
}

\begin{abstract}
This letter presents some further results on the local stability in equilibrium for Internet congestion control algorithm proposed by Low et al. (IEEE/ACM Transactions on Networking, 1999). The propagation delay $d(t)$ is assumed to be time-varying and have maximum and minimum delay bounds (i.e. $d_{m} \leq d(t) \leq d_{M}$ ), which is more general than the assumption $(0<d(t) \leq m)$ made in Long et al.'s work (IEEE Communications Letters, 2003). It is proved that the stability conditions for the Internet congestion control algorithm obtained in Long et al.'s work are in fact dependent on the delay interval $\left(d_{M}-d_{m}\right)$. Moreover, some new stability conditions are proposed, which are less conservative than Long et al.'s results. The proposed linear matrix inequality based stability conditions can be solved by using standard numerical software. These stability conditions provide a method for selecting the parameters in REM algorithm that ensure stability.
\end{abstract}

Index Terms-Congestion control algorithm, linear matrix inequality, time-varying propagation delay, local stability, REM.

\section{INTRODUCTION}

$\mathbf{I}$ T IS well known that the transfer control protocol (TCP) algorithm executed at sources and the active queue management (AQM) algorithm executed at links (routers) determine the equilibrium and dynamics of an IP network, such as the Internet. Therefore, the TCP/AQM pair plays an important role in controlling congestion, which can be interpreted as a distributed algorithm carried out by sources and links over the network to maximize aggregate source utility subject to capacity constraints [7]. As a new AQM, random exponential marking (REM) is proposed in [1], [6], which includes two new ides: (1) The equilibrium value of congestion measure and that of the performance is decoupled such that high utilization with low loss and delay in equilibrium is achieved; (2) By using a probabilistic marking function that is exponential in the link congestion measure, the end-to-end marking probability observed at a source is exponential in the path congestion measure. It has been well demonstrated in [1] via simulations that REM has significant performance advantage.

Dynamics is an important property of any algorithm. Although REM has particular advantage in achieving desirable equilibrium properties (high link utilization with little loss or

Manuscript received September 24, 2004. The associate editor coordinating the review of this letter and approving it for publication was Dr. Nikos Nikolaou. This work was partially supported HKU CRCG 10205251.

H. Gao and C. Wang are with the Harbin Institute of Technology, Harbin, China.

J. Lam is with the Dept. of Mechanical Engineering, University of Hong Kong (e-mail: james.lam@hku.hk).

X. Guan is with Yanshan University, Qinhuangdao, China

Digital Object Identifier 10.1109/LCOMM.2005.05029. queuing delay), its dynamics was not given explicit consideration. Very recently, the stability of REM has drawn much attention from researchers working in related area and a few important results have been reported. These works can be generally divided into two categories according to whether they have taken the propagation delay into consideration. When the delay is absent, the global stability of REM is investigated in [8], [9] by using different approaches. When time delay is considered, [10] derives local stability conditions for REM for a discrete-time multi-link model when all sources have the same delay of one or two-step discrete time. In addition, [5] presents a linear matrix inequality (LMI) based approach to analyze the stability of REM. These conditions provide a guideline for setting REM parameters that ensure stability.

Following the work of Long et al. [5], in this letter we present some further results on the local stability of REM. The propagation delay $d(t)$ is assumed to be time-varying and have maximum and minimum delay bounds (that is, $d_{m} \leq$ $\left.d(t) \leq d_{M}\right)$, which is more general than the assumption $(0<$ $d(t) \leq m)$ made in [5]. It is proved that the stability conditions proposed in Long et al.'s work are actually dependent on the delay interval $\left(d_{M}-d_{m}\right)$. Moreover, some new stability conditions are proposed, which are less conservative than those presented in [5]. The proposed stability conditions are all expressed as LMI-based conditions, which can be solved by using available techniques in standard numerical software [4]. If these conditions are feasible, REM parameters can be readily constructed.

Due to space limitation, in this letter we only consider the single-source algorithm. However, extension to multi-source algorithm can be performed in a trivial manner.

Notations: The superscript " $T$ " stands for matrix transposition; the notation $P>0$ means that $P$ is real symmetric and positive definite; $I$ and 0 represent identity matrix and zero matrix. In symmetric block matrices or long matrix expressions, we use an asterisk $(*)$ to represent a term that is induced by symmetry. Matrices, if their dimensions are not explicitly stated, are assumed to be compatible for algebraic operations.

\section{Model And Existing Result}

Based on the dual model proposed by [1], the network model can be given by the following nonlinear discrete timedelay systems:

$$
\begin{aligned}
& y(t)=x\left(t-d^{\rightarrow}(t)\right) \\
& q(t)=p\left(t-d^{\leftarrow}(t)\right)
\end{aligned}
$$




$$
\begin{aligned}
x(t) & =f(q(t)) \\
p(t+1) & =\left[p(t)+\gamma\left(\alpha\left(b(t)-b^{*}\right)+y(t)-c\right)\right]^{+} \\
b(t+1) & =[b(t)+y(t)-c]^{+}
\end{aligned}
$$

By assuming $d(t)=d^{\rightarrow}(t)+d^{\leftarrow}(t)$, in equilibrium $y^{*}=c$ and $p^{*}>0$, the above nonlinear model is linearized as the following linear discrete time-delay system [5]:

$$
\mathcal{X}(t+1)=\mathcal{A X}(t)+\mathcal{B} \mathcal{X}(t-d(t))
$$

where

$$
\begin{aligned}
\mathcal{X}(t) & =\left[\begin{array}{c}
\bar{p}(t) \\
\bar{b}(t)
\end{array}\right]=\left[\begin{array}{c}
p(t)-p^{*} \\
b(t)-b^{*}
\end{array}\right], \quad \mathcal{A}=\left[\begin{array}{ll}
1 & \eta \\
0 & 1
\end{array}\right], \\
\mathcal{B} & =\left[\begin{array}{cc}
\gamma f^{\prime}\left(p^{*}\right) & 0 \\
f^{\prime}\left(p^{*}\right) & 0
\end{array}\right], \quad \eta=\gamma \alpha
\end{aligned}
$$

In [5], the time-varying delay is assumed to be $1 \leq d(t) \leq$ $m$. Then, according to Lemma 1 in [5], the asymptotic stability of (2) is expressed in the following lemma.

Lemma 1: [5] System (2) is asymptotically stable if there exist matrices $P>0, Q>0$ satisfying

$$
-P+\mathcal{A}^{T} P \mathcal{A}+\mathcal{A}^{T} P \mathcal{B}\left(Q-\mathcal{B}^{T} P \mathcal{B}\right)^{-1} \mathcal{B}^{T} P \mathcal{A}+m Q<0
$$

Since (3) is actually a nonlinear matrix inequality, in order to obtain an LMI-based condition, the following constraints have been imposed:

$$
P=I, \quad Q=\delta I
$$

which leads to the local stability condition for REM given below.

Lemma 2: [5] Suppose the time delay satisfies $1 \leq d(t) \leq$ $m$. The equilibrium of REM is locally asymptotically stable if there exist positive scalars $\gamma, \epsilon, \delta$ and $0<\eta<1$ satisfying

$$
\left[\begin{array}{ccc}
(\epsilon-1) I & 0 & \mathcal{A}^{T} \\
* & -\delta I & \mathcal{B}^{T} \\
* & * & -I
\end{array}\right]<0
$$

Moreover, if we can find a set of feasible solution $\left(\gamma^{*}, \epsilon^{*}, \delta^{*}\right.$, $\left.\eta^{*}\right)$, then $\alpha=\eta^{*} / \gamma^{*}, m=\epsilon^{*} / \delta^{*}$.

\section{MAin Results}

In this letter, we consider a more general assumption on the time delay $d(t)$, that is, the time-varying delay $d(t)$ has maximum and minimum bounds, characterized by

$$
d_{m} \leq d(t) \leq d_{M}
$$

It is worth noting that the assumption $1 \leq d(t) \leq m$ made in [5] can be seen as a particular case of (5) by setting $d_{m}=1$ and $d_{M}=m$. The reason why we consider Assumption (5) is that in practical situations, the minimum delay bound may be known a priori. Taking both the minimum and maximum delay bounds into consideration will in general lead to less conservative stability test, which can be seen more clearly in the following theorem.

Theorem 1: Assume the time delay satisfies (5). System (2) is asymptotically stable if there exist matrices $P>0, Q>0$ satisfying

$$
\begin{aligned}
-P+\mathcal{A}^{T} P \mathcal{A}+\mathcal{A}^{T} P \mathcal{B}\left(Q-\mathcal{B}^{T} P \mathcal{B}\right)^{-1} & \\
& \times \mathcal{B}^{T} P \mathcal{A}+\left(d_{M}-d_{m}+1\right) Q<0
\end{aligned}
$$

Proof. Choose the Lyapunov function as

$$
\begin{aligned}
V(t) & =V_{1}+V_{2}+V_{3} \\
V_{1} & =\mathcal{X}^{T}(t) P \mathcal{X}(t), \quad V_{2}=\sum_{i=t-d(t)}^{t-1} \mathcal{X}^{T}(i) Q \mathcal{X}(i), \\
V_{3} & =\sum_{j=-d_{M}+2}^{-d_{m}+1} \sum_{i=t+j-1}^{t-1} \mathcal{X}^{T}(i) Q \mathcal{X}(i)
\end{aligned}
$$

where $P$ and $Q$ are positive definite matrices to be determined. Define $\Delta V=V(t+1)-V(t)$, then along the solution of (2) we have

$$
\begin{aligned}
\Delta & V_{1}=\mathcal{X}^{T}(t+1) P \mathcal{X}(t+1)-\mathcal{X}^{T}(t) P \mathcal{X}(t) \\
\Delta V_{2}= & \sum_{i=t-d(t+1)+1}^{t} \mathcal{X}^{T}(i) Q \mathcal{X}(i)-\sum_{i=t-d(t)}^{t-1} \mathcal{X}^{T}(i) Q \mathcal{X}(i) \\
= & \mathcal{X}^{T}(t) Q \mathcal{X}(t)-\mathcal{X}^{T}(t-d(t)) Q \mathcal{X}(t-d(t)) \\
& +\sum_{i=t-d(t+1)+1}^{t-1} \mathcal{X}^{T}(i) Q \mathcal{X}(i)-\sum_{i=t-d(t)+1}^{t-1} \mathcal{X}^{T}(i) Q \mathcal{X}(i) \\
\leq & \mathcal{X}^{T}(t) Q \mathcal{X}(t)-\mathcal{X}^{T}(t-d(t)) Q \mathcal{X}(t-d(t)) \\
& +\sum_{i=t-d_{M}+1}^{t-d_{m}} \mathcal{X}^{T}(i) Q \mathcal{X}(i)
\end{aligned}
$$$$
\Delta V_{3}=\left(d_{M}-d_{m}\right) \mathcal{X}^{T}(t) Q \mathcal{X}(t)-\sum_{i=t-d_{M}+1}^{t-d_{m}} \mathcal{X}^{T}(i) Q \mathcal{X}(i)
$$

Therefore, we have

$$
\Delta V \leq \lambda^{T}(t) \Omega \lambda(t)
$$

where

$$
\begin{aligned}
\Omega & =\left[\begin{array}{cc}
\mathcal{A}^{T} P \mathcal{A}-P+\left(d_{M}-d_{m}+1\right) Q & \mathcal{A}^{T} P \mathcal{B} \\
* & \mathcal{B}^{T} P \mathcal{B}-Q
\end{array}\right], \\
\lambda(t) & =\left[\begin{array}{c}
\mathcal{X}(t) \\
\mathcal{X}(t-d(t))
\end{array}\right]
\end{aligned}
$$

By Schur complement, (6) is equivalent to $\Omega<0$, which guarantees $\Delta V<0$ for all nonzero $\mathcal{X}(t)$. Therefore, we can conclude from the standard Lyapunov stability theory that system (2) is asymptotically stable for all time-varying delays satisfying $d_{m} \leq d(t) \leq d_{M}$.

An interesting feature of Theorem 1 lies in the fact that although the derivation process is quite different, the matrix inequality presented in Theorem 1 takes a very similar form as that in Lemma 1 except that $m$ in (3) is replaced by $\left(d_{M}-\right.$ $d_{m}+1$ ). If we set $d_{m}=1$ and $d_{M}=m$ in (6), (6) is exactly the same as (3). In this sense, Theorem 1 generalizes Lemma 1 to a more general case. Another interesting feature of Theorem 1 is that condition (3) is actually dependent only on the varying range of the time delay, that is, $d_{M}-d_{m}$. In other words, if we can find feasible solutions according to Theorem 1 for $d_{m} \leq d(t) \leq d_{M}$, we can also find feasible solutions for $n+\bar{d}_{m} \leq d(\bar{t}) \leq n+d_{M}$ where $n$ is a positive integer.

Similar to Lemma 1, the condition in Theorem 1 is also a nonlinear matrix inequality. It is noted that if we still impose the constraint in (4), we will obtain a stability condition for 
REM similar to Lemma 2. However, the method used in [5] to obtain Lemma 2 deserves some remarks:

1) Constraint (4) is very strong, to obtain less conservative stability result we need to seek different techniques;

2) The obtained delay bound from Lemma 2 is $m=\epsilon^{*} / \delta^{*}$. It is noted that $\epsilon^{*}$ and $\delta^{*}$ are generally real scalars, therefore the obtained $m$ is not guaranteed to be integers, which seems unreasonable and restrictive. In fact, we could leave the delay bound $m$ intact in the matrix inequality, and then use a line search method to find the maximum bound $m$.

In the following, we will establish LMI-based stability conditions for REM based on Theorem 1. First by Schur complement [2], (6) is equivalent to

$$
\left[\begin{array}{ccc}
-P^{-1} & \mathcal{A} & \mathcal{B} \\
* & -P+\left(d_{M}-d_{m}+1\right) Q & 0 \\
* & * & -Q
\end{array}\right]<0
$$

Then, we readily have the following theorem.

Theorem 2: Suppose the time delay satisfies $d_{m} \leq d(t) \leq$ $d_{M}$. The equilibrium of REM is locally asymptotically stable if there exist matrices $L>0, P>0, Q>0, \gamma$ and $0<\eta<1$ satisfying

$$
\left[\begin{array}{ccc}
-L & \mathcal{A} & \mathcal{B} \\
* & -P+\left(d_{M}-d_{m}+1\right) Q & 0 \\
* & * & -Q
\end{array}\right]=0
$$

Moreover, if we can find a set of feasible solution $\left(L^{*}, P^{*}\right.$, $\left.Q^{*}, \gamma^{*}, \eta^{*}\right)$, then $\alpha=\eta^{*} / \gamma^{*}$.

Theorem 2 presents a stability condition for REM, which is equivalent to (6). One may argue that the conditions in Theorem 2 are not strict LMI conditions, however, such conditions are not difficult to solve by using the cone complementarity linearization (CCL) method [3].

Obviously, if we impose the following constraints on the matrix variables in Theorem 1 such that

$$
P=\left[\begin{array}{ll}
1 & 0 \\
0 & p
\end{array}\right], \quad Q=\left[\begin{array}{ll}
q_{1} & q_{2} \\
q_{2} & q_{3}
\end{array}\right]
$$

then by substituting the matrix variables defined in (14) into (11) together with a congruence transformation, we readily obtain the following theorem.

Theorem 3: Suppose the time delay satisfies $d_{m} \leq d(t) \leq$ $d_{M}$. The equilibrium of REM is locally asymptotically stable if there exist scalars $p, q_{1}, q_{2}, q_{3}, \gamma$ and $0<\eta<1$ satisfying

$$
\left[\begin{array}{cccccc}
-1 & 0 & 1 & \eta & \gamma f^{\prime}\left(p^{*}\right) & 0 \\
* & -p & 0 & p & p f^{\prime}\left(p^{*}\right) & 0 \\
* & * & -1+\Delta q_{1} & \Delta q_{2} & 0 & 0 \\
* & * & * & -p+\Delta q_{2} & 0 & 0 \\
* & * & * & * & -q_{1} & -q_{2} \\
* & * & * & * & * & -q_{3}
\end{array}\right]<0
$$

where $\Delta \triangleq d_{M}-d_{m}+1$. Moreover, if we can find a set of feasible solution $\left(p^{*}, q_{1}^{*}, q_{2}^{*}, q_{3}^{*}, \gamma^{*}, \eta^{*}\right)$, then $\alpha=\eta^{*} / \gamma^{*}$.

It is noted that for given $\Delta$, condition (15) is an LMI condition. By noting that $d_{M}$ and $d_{m}$ are positive integers, it is not difficult to find the maximum delay interval $\Delta$ by performing a line search. It is worth noting that since the constraint (14) is less stringent than (4), condition (15) is less conservative than Lemma 2 due to the fact that it can search in a larger variable space.

Finally, to apply the above derived stability conditions to the selection of parameters $\gamma$ and $\alpha$ in model (1) which are essential to the local stability. The following procedure is useful for this purpose:

1) Given model (1), derive its linearized model (2);

2) By solving the LMI-based conditions in Theorem 2 or Theorem 3, obtain the feasible parameters $\gamma^{*}$ and $\eta^{*}$;

3) Calculate the parameters for model (1): $\gamma=\gamma^{*}, \alpha=$ $\eta^{*} / \gamma^{*}$.

\section{CONCLUSIONS}

This letter has presented several new stability conditions for network rate control in the presence of time-varying communication delays. Upon relaxing the assumption on the time-varying delay $(1 \leq d(t) \leq m)$ as used in previous works to a more general case $\left(d_{m} \leq d(t) \leq d_{M}\right)$, several improved stability conditions are provided, which are theoretically shown to be less conservative than the existing ones. All the conditions can be tested by using LMI or LMI-based algorithms in standard numerical software. Due to space limit, stability conditions are only presented for the single-source algorithm. However, the idea behind this letter can be easily extended to the multi-source case.

\section{REFERENCES}

[1] S. Athuraliya, S. H. Low, V. H. Li, and Q. Yin, "REM: active queue management," IEEE Network, vol. 15, pp. 48-53, May/June 2001.

[2] S. Boyd, L. El Ghaoui, E. Feron, and V. Balakrishnan, Linear Matrix Inequalities in Systems and Control Theory. Philadelphia, PA: SIAM, 1994.

[3] L. El Ghaoui, F. Oustry, and M. Ait Rami, "A cone complementarity linearization algorithm for static output-feedback and related problems," IEEE Trans. Automat. Control, vol. 42, pp. 1171-1176, Aug. 1997.

[4] P. Gahinet, A. Nemirovskii, A. J. Laub, and M. Chilali, LMI Control Toolbox User's Guide. Natick, MA: The Math. Works Inc., 1995.

[5] C. N. Long, J. Wu, and X. P. Guan, "Local stability of REM algorithm with time-varying delays," IEEE Commun. Lett., vol. 7, pp. 142-144, Mar. 2003.

[6] S. H. Low and D. E. Lapsley, "Optimization flow control - I: basic algorithm and convergence," IEEE/ACM Trans. Networking, vol. 7, pp. 861-874, June 1999.

[7] S. H. Low, F. Paganini, and J. C. Doyle, "Internet congestion control," IEEE Control Syst. Mag., vol. 22, pp. 28-43, Jan. 2002.

[8] F. Paganini, "On the stability of optimization based flow control," in Proceedings of the American Control Conference, pp. 4689-4694, 2001.

[9] Q. Yin and S. H. Low, "Convergence of REM flow control at a single link," IEEE Commun. Lett., vol. 5 pp. 119-121, Mar. 2001.

[10] Q. Yin and S. H. Low, "On stability of REM algorithm with uniform delay," in Proc. IEEE Global Telecommunications Conference, pp. 26492653, 2002. 